\title{
Overexpressed transcription factor FOXM1 contributes to the progression of colorectal cancer
}

\author{
HONGMEI ZHANG, HUA ZHONG, LEI LI, WANSHENG JI and XIAOQIAN ZHANG
}

Department of Gastroenterology, Affiliated Hospital of Weifang Medical University, Weifang, Shandong 261031, P.R. China

Received February 7, 2015; Accepted November 30, 2015

DOI: $10.3892 / \mathrm{mmr} .2016 .4875$

\begin{abstract}
Forkhead box M1 (FOXM1) is a characteristic proliferation-associated transcription factor, which is overexpressed in various types of human cancer. The aim of the present study was to determine the expression of FOXM1 in a large collection of colorectal cancer (CRC) samples. Between March 2012 and January 2014, 96 patients with histologically diagnosed CRC were recruited into the current study. Using immunohistochemistry, reverse transcription-quantitative polymerase chain reaction and western blotting, mRNA and protein expression levels of FOXM1 in CRC tissue samples were determined. The function of FOXM1 in the CRC cells was evaluated by small interfering RNA-mediated depletion of FOXM1, followed by analyses of cell proliferation and invasion. High levels of staining for FOXM1 were observed in significantly more CRC tissue samples: $85.42 \%(82 / 96)$ of CRC tissue samples compared with $18.75 \%$ (18/96) of adjacent normal mucosa tissue samples. Silencing FOXM1 inhibited the proliferation of LoVo cells, which express a relatively high level of FOXM1, and the invasion and migration of LoVo cells were also markedly suppressed. The data from the present study suggested that the pathogenesis of human CRC may be mediated by FOXM1, and that FOXM1 inhibition may provide a promising therapeutic strategy for CRC.
\end{abstract}

\section{Introduction}

Colorectal cancer (CRC) is a common malignancy worldwide and is a major cause of cancer-associated mortality (1). Despite the marked improvements in surgical and chemoradiotherapeutic techniques that are currently in use, the prognosis of patients with advanced CRC remains poor, and the morbidity remains high (2). In China, the 5-year survival rate of stage IV CRC patients is $8 \%$, and very few survive for 10 years (3).

Correspondence to: Professor Xiaoqian Zhang, Department of Gastroenterology, Affiliated Hospital of Weifang Medical University, 2428 Yuhe Road, Weifang, Shandong 261031, P.R. China E-mail: drzhxq@163.com

Key words: colorectal cancer, forkhead box M1, small interfering RNA, proliferation, invasion, targeted therapy
A notable number of patients with CRC who undergo surgery to remove the cancer develop local recurrence or distant metastasis, resulting in lower survival rates (4). Early diagnosis and surgery are effective ways to cure CRC (5). Rapid integration of novel endoscopic and molecular techniques into clinical practice has established colorectal medicine at the forefront of translational research $(6,7)$, therefore, investigating the molecular mechanisms of CRC and identifying biomarkers for early diagnosis and of prognostic value may aid the selection of suitable therapeutic strategies and enable regular surveillance.

Forkhead box (FOX)M1 is a member of the FOX family of transcription factors $(8,9)$. FOXM1 is key in cell cycle progression, where endogenous FOXM1 expression regulates the transition from the $G_{1}$ to the $S$ phase, as well as the progression to mitosis $(10,11)$. Abnormal upregulation of FOXM1 is observed in a variety of human cancers, including liver, breast, prostate, brain, cervix and lung cancer (12-15). These findings associate FOXM1 with the tumorigenesis and progression of various types of malignancy. However, the role of FOXM1 in the metastatic process of CRC remains to be elucidated. In the present study, the mRNA and protein expression of FOXM1 were investigated in tissue samples from patients with primary CRC using reverse transcription-quantitative polymerase chain reaction (RT-qPCR) and immunohistochemistry, and the current study also aimed to elucidate the effect of FOXM1 on cell invasion and metastasis in CRC cell lines.

\section{Materials and methods}

Tumor tissues. A cohort of 96 patients (57 men and 39 women) with CRC diagnosed between March 2012 and January 2014 were selected. Patient consent and approval from the Ethics Committee of Affiliated Hospital of Weifang Medical University (Weifang, China) were obtained prior to use of these clinical materials for research. Tumor and paired normal samples (10 cm from colorectal tumor) were surgically obtained from these patients. No local or systemic treatment was administered in these patients before surgery. In each selected case, pathological diagnosis was performed in the Department of Pathology, Affiliated Hospital of Weifang Medical University.

Cell culture. The human colorectal cancer cell lines (HCT116, SW480, SW620, LoVo, CaCo-2 and HT-29) were purchased 
A

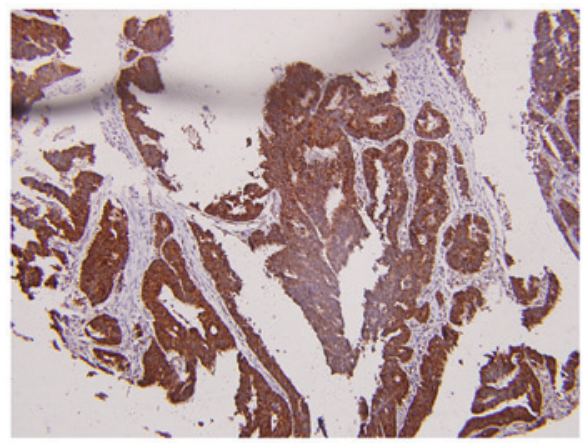

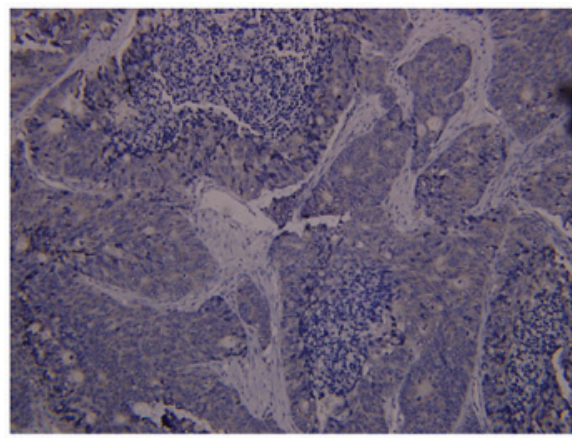

Figure 1. Expression levels of FOXM1 in colorectal cancer. The images represent (A) high and (B) low expression of FOXM1. FOXM1, forkhead box M1. Magnification, $\mathrm{x} 200$.

from the American Type Culture Collection (Manassas, VA, USA). SW620 and SW480 cells were cultured in Leibovitz's L-15 media (Invitrogen; Thermo Fisher Scientific, Inc. Waltham, MA, USA) supplemented with $10 \%$ fetal bovine serum (FBS; Sigma-Aldrich, St. Louis, MO, USA); Caco-2 and HT-29 cells were maintained in RPMI-1640 (Invitrogen; Thermo Fisher Scientific, Inc.) with $10 \%$ FBS, and LoVo cells were cultured in Ham's F-12 Kaighn's medium (Invitrogen; Thermo Fisher Scientific, Inc.) with 10\% FBS.

Immunohistochemistry. Formalin-fixed, paraffin-embedded tissues were cut into 3- $\mu \mathrm{m}$ sections. The tissues were obtained from the Department of Pathology of the Affiliated Hospital of Weifang Medical University, where the formalin fixation process was carried out. The immunohistochemistry steps were performed using streptavidin-biotin peroxidase complex immunostain kit (Wuhan Boster Biological Technology, Ltd., Wuhan, China) according to the manufacturer's protocols. The sections were incubated in a moist chamber with primary rabbit anti-human FOXM1 monoclonal antibody (cat. no., sc-501; dilution, 1:100; Santa Cruz Biotechnology, Inc., Dallas, TX, USA) for $30 \mathrm{~min}$ at room temperature, followed by a horseradish peroxidase-conjugated goat anti-rabbit immunoglobulin $\mathrm{G}$ secondary antibody (cat. no. SA00001-2; (dilution, 1:2,000; Proteintech Group, Inc., Chicago, IL, USA) for $30 \mathrm{~min}$ at room temperature. Rabbit serum (HyClone, Logan, UT, USA) served as a negative control. The images were collected and analyzed using a Leica DC200 image system (Leica Microsystems, Inc., Buffalo Grove, IL, USA).

Immunohistochemical analysis evaluation. Five random microscopic fields (magnification, x200) were examined per slide, and 100 cells were evaluated per field. The expression of FOXM1 was classified into five groups according to the percentage of positively staining cells: $0=$ absent; $1=1-25 \% ; 2=26-50 \%$; $3=51-75 \%$; and $4=\geq 76 \%$. The staining intensity was categorized as follows: $0=$ negative; $1=$ weak; $2=$ moderate; and $3=$ strong. The proportion and intensity scores were subsequently multiplied to obtain a total score. Where the product of multiplication between staining intensity and the percentage of positive cells was $\leq 4$, it was defined as low expression; by contrast, where the overall score was $>4$, it was defined as high expression.

Small interfering RNA (siRNA) transfection. Three FOXM1 siRNAs were designed and synthesized by Qiagen $\mathrm{GmbH}$
(Hilden, Germany) to generate effective FOXM1-siRNA oligonucleotides for gene knockdown studies. An siRNA with the sequence CUCUUCUCCCUCAGAUAUAdTdT was determined to exert the greatest effect on inhibition of FOXM1 expression. The LoVo CRC cells were transfected with FOXM1-siRNA oligonucleotides or control siRNA oligonucleotides (50 nmol/1; Santa Cruz Biotechnology, Inc.) with the use of Lipofectamine ${ }^{\circledR} 2000$ Transfection reagent (Invitrogen; Thermo Fisher Scientific, Inc.) according to the manufacturer's protocols.

$R T-q P C R$ assay. Total RNA was isolated from the cell lines using TRIzol Reagent (Invitrogen; Thermo Fisher Scientific, Inc.). The concentration and quality of the extracted total RNA was determined by measuring the optical density at 260 and $280 \mathrm{~nm}$, and then calculating the OD260:OD280 ratio. Complementary DNA (cDNA) was synthesized from $2 \mu \mathrm{g}$ of total RNA using a Reverse Transcription System kit (Thermo Fisher Scientific, Inc.), according to the manufacturer's protocol. Briefly, the samples were pre-incubated at $70^{\circ} \mathrm{C}$ for $10 \mathrm{~min}$, cooled on ice and then added to a reaction mixture consisting of $10 \mathrm{mmol} / 1$ deoxynucleotide triphosphate, $25 \mathrm{mmol} / \mathrm{l} \mathrm{MgCl} 2,15$ units avian myoblastosis virus reverse transcriptase, $10 \mathrm{X}$ reverse transcription buffer, 0.5 units $\mathrm{RNasin}^{\circledR}$ and $0.5 \mu \mathrm{g}$ oligo-(dT) $)_{15}$ primer (all provided with the Reverse Transcription System kit). A final volume of 201 reaction mixture was incubated successively at $44^{\circ} \mathrm{C}$ for $15 \mathrm{~min}$, $99^{\circ} \mathrm{C}$ for $5 \mathrm{~min}$ and $4^{\circ} \mathrm{C}$ for $5 \mathrm{~min}$. The cDNA was maintained at $20^{\circ} \mathrm{C}$ prior to use. Then, it was reverse-transcribed from $5 \mu \mathrm{g}$ of total RNA. RT-qPCR was performed using a SYBR Master Mix (Takara Bio Inc., Otsu, Japan) on an ABI Prism 7900HT Sequence Detection System (Applied Biosystems; Thermo Fisher Scientific, Inc.). The primer sequences of FOXM1 were as follows: Sense, 5'-GGAGGAAATGCCACACTTAGCG-3'; and reverse, 5'-TAGGACTTCTTGGGTCTTGGGGTG-3' (designed by Guangzhou Ruibo Trading Co., Ltd., Guangzhou, China). The products of PCR were separated by $1.5 \%$ agarose gel electrophoresis, and the expression levels of the mRNA were detected, with GAPDH serving as the control. The primer sequences for GAPDH were as follows: Sense, 5'-GCA GTGGCAAAGTGGAGATT-3'; and reverse, 5'-TGA AGT CGCAGGAGACAACC-3'. The cycling conditions used were as follows: $94^{\circ} \mathrm{C}$ for $2 \mathrm{~min}$ for 2 cycles, followed by 40 cycles of $94^{\circ} \mathrm{C}$ for $15 \mathrm{sec}, 58^{\circ} \mathrm{C}$ for $25 \mathrm{sec}$, and $72^{\circ} \mathrm{C}$ for $30 \mathrm{sec}$. qPCR was conducted on an Applied Biosystems 7500 Real-Time 
A

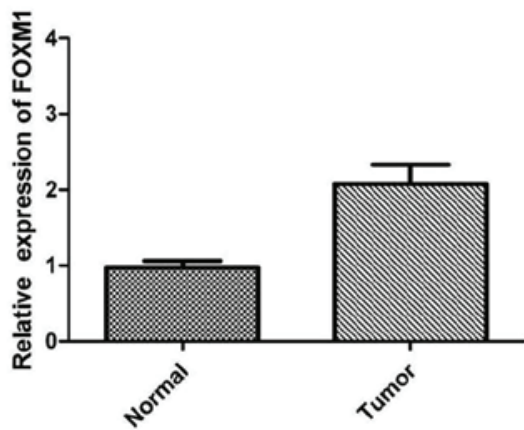

B

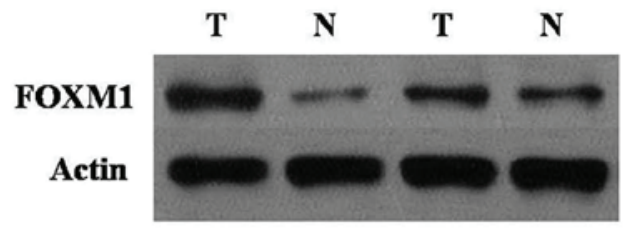

Figure 2. (A) Reverse transcription-quantitative polymerase chain reaction demonstrating increased mRNA expression levels of FOXM1 in CRC and (B) a western blot demonstrating the expression levels of FOXM1 in CRC. CRC, colorectal cancer; FOXM1, forkhead box M1; T, tumor; N, normal.

A

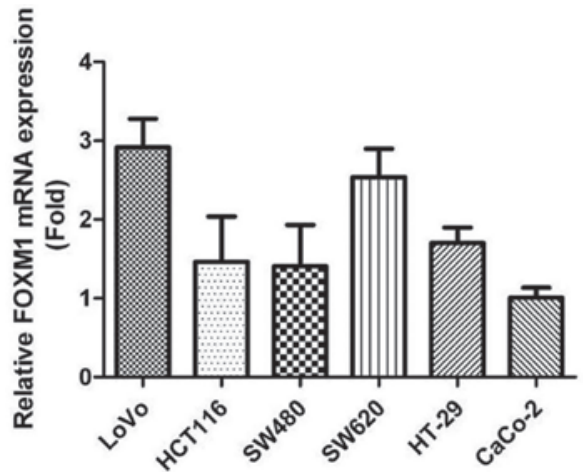

B

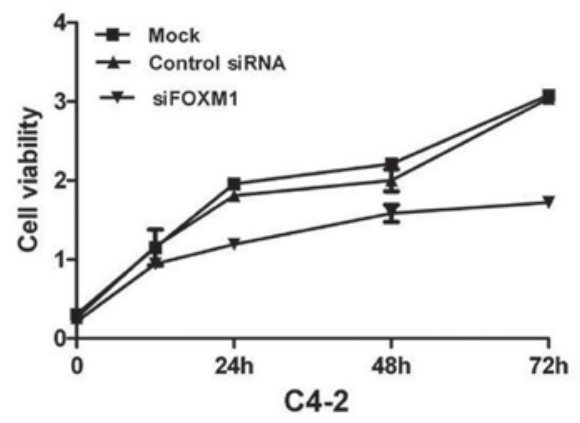

Figure 3. (A) Reverse transcription-quantitative polymerase chain reaction demonstrating the mRNA expression levels of FOXM1 in CRC cells. (B) FOXM1 and knockdown-inhibited cell proliferation of LoVo cells. Viable cells were measured using the 3-(4,5-dimethylthiazol-2-yl)-2,5-diphenyltetrazolium bromide assay. FOXM1, forkhead box M1; mRNA, messenger RNA; siRNA, small interfering RNA.

PCR system (Applied Biosystems; Thermo Fisher Scientific, Inc.). Each experiment was performed three times, each time in duplicate. The relative expression levels of Trop2 mRNA were calculated using the $2^{-\Delta \Delta C t}$ method (16).

Western blot analysis. Whole cell lysates were prepared from the colon cells as described previously (17). Standard western blot analysis of the lysates was conducted with the primary rabbit anti-human FOXM1 monoclonal antibody and a second anti-IgG antibody (GE Healthcare Life Sciences, Chalfont, UK). The membranes were then stripped and blotted, and an anti- $\beta$-actin antibody (Sigma-Aldrich) served as a loading control. Blots were visualized using an enhanced chemiluminescence kit (Santa Cruz Biotechnology., Inc.) according to the manufacturer's protocol, and exposed to film (X-OMAT BT; Kodak, Rochester, NY, USA).

Cell survival assay. The effects of FOXM1 on CRC cell survival were determined using the 3-(4,5-dimethylthiazol-2-yl)-2,5-diphenyltetrazolium bromide (MTT) assay. Four groups of cells were seeded into 96-well plates (Nunc A/S Plastfabrikation, Roskilde, Denmark; $5 \times 10^{3}$ cells/well) and cultured for $120 \mathrm{~h}$. Following treatment, cells were incubated with MTT (20 $\mu \mathrm{l} /$ well; Sigma-Aldrich) at $37^{\circ} \mathrm{C}$ for $4 \mathrm{~h}$, and then $200 \mu$ ldimethyl sulfoxide was added to each well. The absorbance of the cells was measured at a wavelength of $570 \mathrm{~nm}$ using a microplate reader (Bio-Tek Instruments, Inc., Winooski, VT, USA). The percentage of residual cell viability

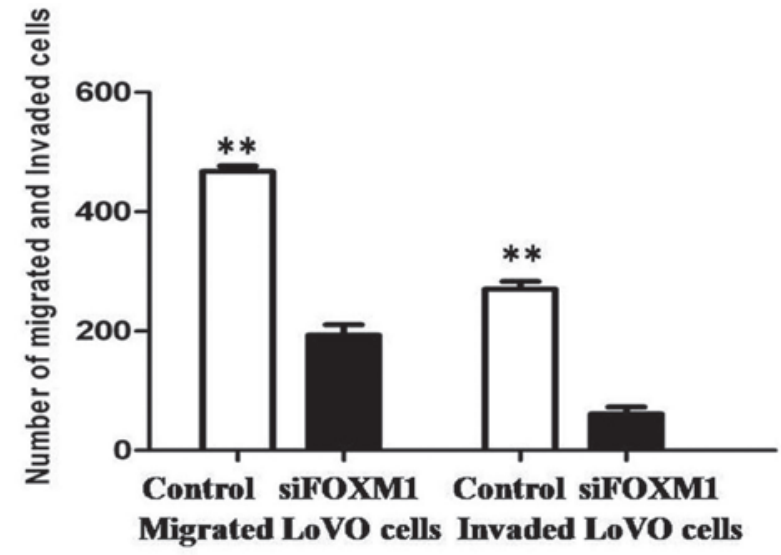

Figure 4. Inhibition of invasion and migration of LoVo Cells by FOXM1 siRNA treatment. ${ }^{* *} \mathrm{P}<0.05$. FOXM1, forkhead box M1; siRNA, small interfering RNA.

was determined by: [(optical density (OD) of experimental group - OD of blank group)/(OD of negative group - OD of blank group)] $\mathrm{x} 100 \%$. The assays were performed three times.

Cell migration assay. Motility in vitro was assessed using Transwell ${ }^{\circledR}$ chambers (Corning Incorporated, Corning, NY, USA). Four groups of cells $\left(5 \times 10^{5}\right)$ were seeded on the upper wells with Ham's F-12K Kaighn's medium. Medium (500 $\mu$ l) with $20 \%$ FBS was plated in the bottom wells as chemoattractants. Following a 48-h incubation, cells were fixed with 
methanol and stained with $1 \%$ crystal violet (Sigma-Aldrich) for $30 \mathrm{~min}$ at $37^{\circ} \mathrm{C}$. Cells on the upper filter of the membranes were wiped, while cells that had penetrated to the lower filter were stained with hematoxylin (Sigma-Aldrich). Cells in 15 randomized fields were counted and images were captured using an inverted microscope (magnification, x50; Olympus BX51; Olympus Corp., Tokyo, Japan).

Statistical analysis. Data analyses were performed using SPSS version 15.0 (SPSS, Inc., Chicago, IL, USA). Patient characteristics were expressed as the mean \pm standard deviation for continuous variables, and as the count and percentage for discrete variables. The data were analyzed using the Pearson's chi-square test and Fisher's exact test, and $\mathrm{P}<0.05$ was considered to indicate a statistically significant difference.

\section{Results}

To investigate the clinical significance of FOXM1 expression in human CRC progression and metastasis, FOXM1 expression was investigated using immunohistochemistry, western blotting and RT-qPCR in 96 CRC tissue samples. As presented in Fig. 1A and B, the expression of FOXM1 was higher in 82 of 96 randomly selected human CRC tissues compared with the adjacent normal mucosa tissues. High expression of FOXM1 was observed in significantly more of the CRC tissue samples [85.42\% (82/96) of the CRC tissues] compared with $18.75 \%$ $(18 / 96)$ of adjacent normal mucosa tissues $(\mathrm{P}<0.001)$. The FOXM1 mRNA expression levels were normalized to GAPDH mRNA and detected by RT-qPCR (Fig. 2A). The results indicated that the relative expression level of FOXM1 mRNA in CRC tissues was significantly higher compared with adjacent normal mucosa tissues $(\mathrm{P}<0.01)$. These results were supported by western blot analysis, and protein expression levels of FOXM1 were significantly higher in CRCs than in adjacent normal mucosa tissues $(\mathrm{P}<0.01)$ (Fig. 2B). These data suggest that, as FOXM1 is overexpressed in CRC tissue samples, it may be important in CRC progression.

RT-qPCR analysis was used to determine the mRNA expression levels of FOXM1 in CRC cell lines. The results indicated that FOXM1 was highly expressed in LoVo cells, so this cell line, among others, was used in the present study (Fig. 3A). To investigate the effects of FOXM1 silencing on cell proliferation, FOXM1 was downregulated in LoVo cells using siRNA against FOXM1 transcripts. The cells were assessed for cell proliferation using the MTT assay, and the results indicated that FOXM1-siRNA-transfected LoVo cells demonstrated a significantly lower proliferation rate compared with the control groups $(\mathrm{P}<0.05)$ (Fig. 3B). The effects of FOXM1 silencing on tumor cell metastasis were also investigated. Cellular invasiveness and migration were analyzed by Transwell ${ }^{\circledR}$ assays, and the migratory and invasive abilities were revealed to be attenuated in FOXM1-siRNA-transfected LoVo cells (Fig. 4).

\section{Discussion}

In developed countries, CRC is the third most commonly diagnosed malignancy, and the second leading cause of cancer-associated mortality (18). A notable number of patients with CRC who undergo surgery to treat the cancer develop local recurrence or distant metastasis, leading to lower survival rates (19); the same stage of the disease may present different clinical courses and have a different prognosis due to intraand intertumor heterogeneity (20). Although the regulation of CRC growth is well understood, the mechanism underlying $\mathrm{CRC}$ proliferation, migration, invasion and metastasis remains to be elucidated. In the present study, a high expression of FOXM1 was observed in significantly more tumor tissue samples $[85.42 \%(82 / 96)$ of the CRC tissues vs. $18.75 \%(18 / 96)$ of adjacent normal mucosa tissues] by immunohistochemical analysis. It was demonstrated that the expression levels of FOXM1 were significantly higher in CRC tissues than in adjacent normal mucosa tissues, at the transcriptional and the translational level. The results of the present study are consistent with previous studies that reported the overexpression of FOXM1 in various human cancers.

FOXM1 is a proliferation-associated transcription factor with important roles in cell proliferation, differentiation and apoptosis (21). It is expressed in actively dividing cells, and is critical for cell cycle progression. It was first identified as a proliferation-specific transcription factor expressed in various tumor cell lines and embryonic tissues (22). Multiple signaling pathways are associated with the FOXM1 signaling pathway, suggesting that FOXM1 is central to tumor aggressiveness (23). Previous studies have demonstrated that FoxM1 is upregulated in a wide variety of malignant tumors (23-26). The current study demonstrated that FOXM1 is highly expressed in tissue samples of patients with CRC. Thus, the biological function of FOXM1 was examined in greater detail via in vitro analysis of the CRC cell lines. The mRNA expression levels of FOXM1 in the CRC cell lines were investigated by RT-qPCR analysis. LoVo cells demonstrated a relatively high FOXM1 expression level, and these were used for further study. siRNA was used to knockdown FOXM1 expression in LoVo cell lines. An impaired proliferation capacity of LoVo cells was observed following FOXM1 knockdown. Downregulation of FOXM1 was also demonstrated to inhibit cell migration and invasion. Thus, the present study suggested that FOXM1 is a potential therapeutic target for the treatment of CRC.

In conclusion, the present study demonstrated the clinical significance of overexpressed FOXM1 in patients with CRC. Results from the current study indicate that FOXM1 may serve as a promising therapeutic target for the inhibition of CRC progression, and that targeting FOXM1 may provide a novel therapeutic strategy for the treatment of CRC.

\section{References}

1. Perencevich M and Stoffel EM: A multidisciplinary approach to the diagnosis and management of multiple colorectal polyps. Gastroenterol Hepatol (NY) 7: 420-423, 2011.

2. Shelton BK: Introduction to colorectal cancer. Semin Oncol Nurs 18 (Suppl 2): S2-S12, 2002.

3. Shimada H, Tanaka K, Endou I and Ichikawa Y: Treatment for colorectal liver metastases: A review. Langenbecks Arch Surg 394: 973-983, 2009.

4. Al-Maghrabi J, Emam E, Gomaa W, Saggaf M, Buhmeida A, Al-Qahtani M and Al-Ahwal M: c-MET immunostaining in colorectal carcinoma is associated with local disease recurrence. BMC Cancer 15: 676, 2015.

5. Jagadish N, Parashar D, Gupta N, Agarwal S, Purohit S, Kumar V, Sharma A, Fatima R, Topno AP, Shaha C and Suri A: A-kinase anchor protein 4 (AKAP4) a promising therapeutic target of colorectal cancer. J Exp Clin Cancer Res 34: 142, 2015. 
6. Fearon ER: Molecular genetics of colorectal cancer. Annu Rev Pathol 6: 479-507, 2011.

7. Boland CR and Goel A: Microsatellite instability in colorectal cancer. Gastroenterology 138: 2073-2087, 2010.

8. Elzagallaai AA, Garcia-Bournissen F, Finkelstein Y, Bend JR, Rieder MJ and Koren G: Severe bullous hypersensitivity reactions after exposure to carbamazepine in a Han-Chinese child with a positive HLA-B*1502 and negative in vitro toxicity assays: Evidence for different pathophysiological mechanisms. J Popul Ther Clin Pharmacol 18: e1-e9, 2011.

9. Raychaudhuri P and Park HJ: FoxM1: A master regulator of tumor metastasis. Cancer Res 71: 4329-4333, 2011.

10. Kalin TV, Ustiyan V and Kalinichenko VV: Multiple faces of FoxM1 transcription factor: Lessons from transgenic mouse models. Cell Cycle 10: 396-405, 2011.

11. Wang IC, Chen YJ, Hughes D, Petrovic V, Major ML, Park HJ, Tan Y, Ackerson T and Costa RH: Forkhead box M1 regulates the transcriptional network of genes essential for mitotic progression and genes encoding the SCF (Skp2-Cks1) ubiquitin ligase. Mol Cell Biol 25: 10875-10894, 2005.

12. Sun H, Teng M, Liu J, Jin D, Wu J, Yan D, Fan J, Qin X, Tang H and Peng Z: FOXM1 expression predicts the prognosis in hepatocellular carcinoma patients after orthotopic liver transplantation combined with the Milan criteria. Cancer Lett 306: 214-222, 2011

13. Yu J, Deshmukh H, Payton JE, Dunham C, Scheithauer BW Tihan T, Prayson RA, Guha A, Bridge JA, Ferner RE, et al: Array-based comparative genomic hybridization identifies CDK4 and FOXM1 alterations as independent predictors of survival in malignant peripheral nerve sheath tumor. Clin Cancer Res 17: 1924-1934, 2011

14. Balli D, Zhang Y, Snyder J, Kalinichenko VV and Kalin TV: Endothelial cell specific deletion of transcription factor FoxM1 increases urethane-induced lung carcinogenesis. Cancer Res 71: 40-50, 2011.

15. Calvisi DF, Pinna F, Ladu S, Pellegrino R, Simile MM, Frau M, De Miglio MR, Tomasi ML, Sanna V, Muroni MR, et al: Forkhead box M1B is a determinant of rat susceptibility to hepatocarcinogenesis and sustains ERK activity in human HCC. Gut 58: 679-687, 2009.

16. Livak KJ and Schmittgen TD: Analysis of relative gene expression data using real-time quantitative PCR and the 2(-Delta Delta C(T)) Method. Methods 25: 402-408, 2001.
17. Chen GQ, Tang CF, Shi XK, Lin CY, Fatima S, Pan XH, Yang DJ, Zhang G, Lu AP, Lin SH and Bian ZX: Halofuginone inhibits colorectal cancer growth through suppression of Akt/mTORC1 signaling and glucose metabolism. Oncotarget 6: 24148-24162, 2015

18. Rasool S, Kadla SA, Rasool V and Ganai BA: A comparative overview of general risk factors associated with the incidence of colorectal cancer. Tumour Biol 34: 2469-2476, 2013.

19. Van Cutsem E, Nordlinger B, Adam R, Köhne CH, Pozzo C, Poston G, Ychou M and Rougier P; European Colorectal Metastases Treatment Group: Towards a pan-European consensus on the treatment of patients with colorectal liver metastases. Eur J Cancer 42: 2212-2221, 2006.

20. Poston GJ, Figueras J, Giuliante F, Nuzzo G, Sobrero AF, Gigot JF, Nordlinger B, Adam R, Gruenberger T, Choti MA, et al: Urgent need for a new staging system in advanced colorectal cancer. J Clin Oncol 26: 4828-4833, 2008.

21. Lam EW, Brosens JJ, Gomes AR and Koo CY: Forkhead box proteins: Tuning forks for transcriptional harmony. Nat Rev Cancer 13: 482-95, 2013.

22. Cai Y, Balli D, Ustiyan V, Fulford L, Hiller A, Misetic V, Zhang Y, Paluch AM, Waltz SE, Kasper S and Kalin TV: Foxm1 expression in prostate epithelial cells is essential for prostate carcinogenesis. J Biol Chem 288: 22527-22541, 2013.

23. Fan Q, Cai Q and Xu Y: FOXM1 is a downstream target of LPA and YAP oncogenic signaling pathways in high grade serous ovarian cancer. Oncotarget 6: 27688-27699, 2015.

24. Yu G, Zhou A, Xue J, Huang C, Zhang X, Kang SH, Chiu WT, Tan C, Xie K, Wang J and Huang S: FoxM1 promotes breast tumorigenesis by activating PDGF-A and forming a positive feedback loop with the PDGF/AKT signaling pathway. Oncotarget 6: 11281-11294, 2015.

25. Consolaro F, Basso G, Ghaem-Magami S, Lam EW and Viola G: FOXM1 is overexpressed in B-acute lymphoblastic leukemia (B-ALL) and its inhibition sensitizes B-ALL cells to chemotherapeutic drugs. Int J Oncol 47: 1230-1240, 2015.

26. Barger CJ, Zhang W, Hillman J, Stablewski AB, Higgins MJ, Vanderhyden BC, Odunsi K and Karpf AR: Genetic determinants of FOXM1 overexpression in epithelial ovarian cancer and functional contribution to cell cycle progression. Oncotarget 6 : 27613-27627, 2015. 